\title{
Two-Dimensional Adaptive Block Kalman Filtering of SAR Imagery
}

\author{
Mahmood R. Azimi-Sadjadi, Senior Member, IEEE, and Sami Bannour, Student Member, IEEE
}

\begin{abstract}
Speckle effects are commonly observed in synthetic aperture radar (SAR) imagery. In airborne SAR systems the effect of this degradation reduces the accuracy of detection substantially. Thus, the elimination of this noise is an important task in SAR imaging systems. In this paper a new method for speckle noise removal is introduced using 2-D adaptive block Kalman filtering (ABKF). The image process is represented by an autoregressive (AR) model with nonsymmetric half-plane (NSHP) region of support. New 2-D Kalman filtering equations are derived which take into account not only the effect of speckles as a multiplicative noise but also those of the additive receiver thermal noise and the blur. This method assumes local stationarity within a processing window, whereas the image can be assumed to be globally nonstationary. A recursive identification process using the stochastic Newton approach is also proposed which can be used on-line to estimate the filter parameters based upon the information within each new block of the image. Simulation results on several images are provided to indicate the effectiveness of the proposed method when used to remove the effects of speckle noise as well as that of the additive noise.
\end{abstract}

\section{INTRODUCTION}

QYNTHETIC aperture radar (SAR) systems are used in Mmany applications including space imagery. The popularity can be attributed to a great extent to their alongtrack linear resolution characteristic, which is independent of range. However, since SAR systems rely upon coherence properties of the scattered signals they are highly susceptible to speckles. Speckles appear in SAR images as tiny spots with varying intensities. The source of this noise is attributed to random interference of wavelets scattered by the microscopic fluctuations of the object surface within one resolution element. The presence of the speckle noise in an imaging system reduces the resolution by as much as a factor of seven and is particularly severe in low contrast images. Therefore, the suppression of this noise is an important consideration in the design of coherent imaging systems. Speckles have the characteristics of a random multiplicative noise in the sense that the terrain backscatter (the desired image) is multiplied by a stationary random process which represents the effects of coherent fading [1]. This modeling is only valid when the bandwidth of the signal is small compared with

Manuscript received August 2, 1988; revised April 15, 1991.

The authors are with the Department of Electrical Engineering, Colorado State University, Fort Collins, CO 80523

IEEE Log Number 91013480. that of the imaging device [2]. Another constraint which makes the restoration process in the presence of speckle noise different from the standard minimum mean squared error (MMSE) filtering scheme is that for radar, the signal is nonstationary since the mean backscatter changes with the type of target being sensed, despite the fact that the noise can be modeled as stationary.

Basically SAR speckle reduction techniques fall into two categories. The techniques in the first category involve noncoherent integration, which aims at improving the appearance of SAR images by averaging several uncorrelated frames from nonoverlapping spectra [3], [4]. This averaging process increases the SNR by a factor of $M^{1 / 2}$ where $M$ is the number of frames. While these methods are effective for speckle reduction, they require multiple frames of the speckled images and do not take into account a statistical model for the image. Moreover, they fail to consider the effects of other degradations such as the receiver thermal noise, the radiometric quantization noise, which are additive, and blur. In recent years, application of digital image restoration techniques to SAR image processing has attracted considerable attention. These, known as post-image formation methods, involve developing statistical models for the signal (terrain backscatter) and the noise and then making use of either the standard frequency domain methods such as Wiener filtering [5]-[7] or recursive filtering using Kalman filters [8]. The major disadvantage of the former class of techniques is that they are inherently space invariant and do not account for the nonstationarity in the image. Moreover, they are not effective when the image is corrupted by multiple degradations. The recursive filtering techniques may also suffer from the same deficiency if the image is assumed to be globally stationary. However, the assumption of stationarity may be applied to small regions. In this case, it is necessary to adapt the filter parameters to the local changes in the image.

Several nonlinear and adaptive image restoration techniques have been proposed over the past few years. Anderson and Netravali [9] used a subjective error criterion based on human visual system models and derived a nonrecursive filter. Abramatic and Silverman [10] generalized this method and related it to the classical Wiener filter. Ingle and Woods [11] proposed a composite image model using the reduced update Kalman filter (RUKF). This method which takes into account the specific corre- 
lation directions results in a bank of Kalman filters running in parallel. Rajala et al. [12] proposed an adaptive Kalman filtering scheme based upon partitioning the image into disjoint regions according to the local spatial activity determined by the directional derivative information. Other features of this method include the use of nearest neighbor algorithm to determine the best previous states and a 2-D interpolation scheme to improve the estimates of the initial states in each region. Another approach, which is based upon continuous adaptation of filter parameters during the scanning process in a local window, has been proposed by Lee [13]. Later, he used this approach for speckle noise removal using Sigma filters [14]. However, this approach does not consider a model for the image process. Kuan et al. [15] extended Lee's local statistics algorithm to the case of nonstationary mean-nonstationary variance (NMNV) image model. Frost et al. [16] addressed the problem of optimum MMSE filtering of images in the presence of multiplicative noise with particular application to SAR. By using the locally estimated parameter values, the filter is made adaptive. However, it is shown that [17] their model is not optimum in the MSE sense. More recently, Kuan et al. [18] derived a local MMSE (LMMSE) filter based upon a NMNV image model which accounts for the correlation properties of the speckle noise. Azimi-Sadjadi [19], [20] introduced a 2-D adaptive block Kalman filter (ABKF) which can be used to remove the effects of multiple degradations from SAR images.

In this paper a new method for speckle noise reduction is proposed. An adaptive block Kalman filtering ( $A B K F$ ) scheme is developed which takes into account the effects of multiple degradations due to speckle noise, additive receiver thermal noise, and linear space invariant (LSI) blur. For an autoregressive (AR) image model with nonsymmetric half plane (NSHP) region of support a new 2-D block state-space model is derived in Section II in which the states propagate in two directions. The degradation process due to speckle, additive noise, and blur is also modeled by a block equation in Section III. In Section IV, these equations are combined to form a complete block dynamic model for the entire process. For this model a set of 2-D Kalman filter equation is derived which accounts for the effects of multiple degradations. A new 2-D recursive identification procedure is developed in Section $\mathrm{V}$ which can be used on-line to adjust the AR model parameters in each processing window. In Section VI the simulation results on an SAR image degraded by speckle noise and additive noise are presented and a comparison between the proposed scheme and other locally adaptive methods is made.

\section{A 2-D Block Diagonal Model for the Image PROCESS}

Consider an image of size $\mathrm{N} \times \mathrm{N}$ which is scanned row by row from left to right and top to bottom. The image is assumed to be represented by a zero mean Markov field and modeled by an $M \times M$ order causal $A R$ model given by

$$
x(m, n)=\sum_{p, q \in W} a_{p, q} x(m-p, n-q)+u(m, n)
$$

where $x(m, n)$ represents the pixel intensity at location ( $m$, $n) ; u(m, n)$ is a white noise sequence which drives the process; and $a_{p, q}$ 's are the reflection coefficients of the AR model. For an AR model with NSHP region of support, the prediction window $W$ is given by

$$
\begin{gathered}
W=\{p, q: 1 \leq p \leq M,-M \leq q \leq M, \quad \text { and } \\
p=0,1 \leq q \leq M\} .
\end{gathered}
$$

The region of support of this model is shown in Fig. 1 . The statistics of the driving process $u(m, n)$ are

$$
\begin{aligned}
E[u(m, n)] & =0 \\
E[u(m+k, n+l) u(m, n)] & =\boldsymbol{\sigma}_{u}^{2} \boldsymbol{\delta}(k, l)
\end{aligned}
$$

where $\sigma_{u}^{2}$ is the variance of the error $u(m, n) ; \delta(k, l)$ is the Kronecker delta function and $E$ is the expectation operator. The orthogonality property of the minimum variance estimator gives

$$
E[u(m, n) x(m-p, n-q)]=0 \quad(p, q) \in \boldsymbol{W} .
$$

The parameters of the AR model, i.e., $a_{p, q}$ 's can be evaluated based upon the global estimates of the autocorrelation sequence of the image on a finite window. Let the autocorrelation sequence of the image $\{x(m, n)\}$ be denoted by

$$
r_{x}(k, l):=E[x(m, n) x(m-k, n-l)]
$$

then the normal equation obtained from (1) is

$$
r_{x}(k, l)=\sum_{p, q \in W} a_{p, q} r_{x}(k-p, l-q)+\boldsymbol{\sigma}_{u}^{2} \boldsymbol{\delta}(k, l)
$$

Now, the parameters of the AR model, $a_{p, q}$ 's and the variance of the driving noise, $\sigma_{u}^{2}$, can be obtained [21], [22] by solving the system of normal equations (6). Having evaluated these parameters, the AR model (1) can then be arranged into a 2-D block recursive form. The direct extension of the block processing method [23] to AR models with NSHP region of support leads to structures which exhibit noncausality and hence cannot be implemented recursively. Gnanasekaran [24] and Lee [25] proposed different 2-D block structures for models with NSHP and SHP regions of support. However, their block structures are computationally laborious and incompatible with the structure of the 2-D Kalman filter. In this section a new 2-D block recursive structure for AR models with NSHP region of support is derived based upon the idea of arranging the pixels in a diagonal block form. We begin by defining the $(i, j)$ th diagonal block of size $K \times L$ by

$X_{i, j}=\left[X_{i K}(j L+L-1) X_{i K}(j L+L-2) \cdots X_{i K}(j L)\right]^{\prime}$ 
where

$$
\begin{gathered}
X_{i K}(j L+l)=[x(i K, j L+l) x(i K+1, \\
\quad j L+l-M) \cdots x(i K+K-1, \\
j L+l-(K-1) M)] .
\end{gathered}
$$

The arrangement of pixels in a diagonal block is shown in Fig. 2. From an implementation point of view, the optimal choice for the length of each diagonal, i.e., $K$ is found to be $(M+1)$. It is also observed that the minimum width for each block is $L=M(M+1)$. These issues will be discussed later in this section. Now, writing the AR equation for each pixel in a diagonal block $(i, j)$ and arranging them into a block vector yields the following 2 $\mathrm{D}$ block equation which is somewhat similar to that of the quarter plane case [23].

$$
\begin{array}{r}
\mathrm{A}_{00} X_{i, j}+\mathrm{A}_{01} X_{i, j-1}+\mathrm{A}_{10} X_{i-1 . j} \\
+\mathrm{A}_{1-1} X_{i-1, j+1}+U_{i, j}=0
\end{array}
$$

or

$$
\begin{gathered}
X_{i, j}=\mathrm{B}_{01} X_{i, j-1}+\mathrm{B}_{10} X_{i-1, j}+\mathrm{B}_{1-1} X_{i-1, j+1}+\mathrm{B}_{00} U_{i, j} \\
i \in[0,(\lceil N / K\rceil-1)], j \in[0,\lceil N / L\rceil]
\end{gathered}
$$

where \lceil\rceil represents the ceiling operator

$$
\begin{aligned}
& \mathrm{B}_{00}=-\mathrm{A}_{00}^{-1} \\
& \mathrm{~B}_{01}=\mathrm{B}_{00} \mathrm{~A}_{01}, \quad \mathrm{~B}_{10}=\mathrm{B}_{00} \mathrm{~A}_{10}, \quad \mathrm{~B}_{1-1}=\mathrm{B}_{00} \mathrm{~A}_{1-1}
\end{aligned}
$$

and $U_{i, j}$ represents a block of white noise sequence $\{u(m$, $n)\}$ defined in a manner similar to $(7)$. The matrices $A_{i j}$, $s$ that are either lower or upper triangular block Toeplitz are defined as

$$
\begin{aligned}
\mathrm{A}_{00}= & {\left[\begin{array}{cccc}
\mathrm{A}_{0} & \mathrm{~A}_{1} & \cdots & \mathrm{A}_{L-1} \\
0 & \mathrm{~A}_{0} & \cdots & \mathrm{A}_{L-2} \\
\vdots & \ddots & \ddots \\
\bigcirc & & \mathrm{A}_{0}
\end{array}\right]_{K L \times K L} } \\
\mathrm{~A}_{01} & =\left[\begin{array}{ccc}
\mathrm{A}_{L} & & \bigcirc \\
\mathrm{A}_{L-1} & \mathrm{~A}_{L} & \\
\vdots & \vdots & \ddots \\
\mathrm{A}_{1} & \mathrm{~A}_{2} & \mathrm{~A}_{L}
\end{array}\right]_{K L \times K L}
\end{aligned}
$$

Matrices $A_{1-1}$ and $A_{10}$ are similarly defined in which $A_{i}$ 's are replaced by $A_{i}^{\prime}$ 's. The block matrices $A_{i}$ 's and $A_{i}^{\prime}$ 's are themselves either lower or upper triangular Toeplitz given by

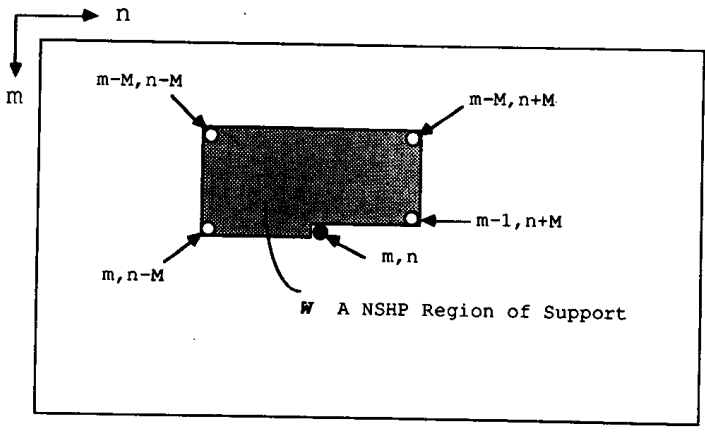

Fig. 1. An NSHP region of support of the AR model.

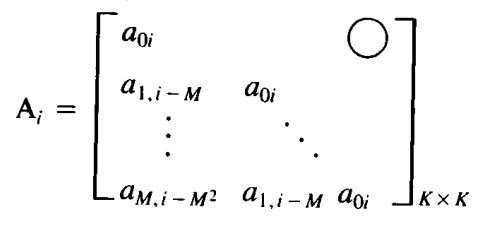

$\mathbf{A}_{i}^{\prime}=\left[\begin{array}{cccc}0 & a_{M, i-M^{2}} & a_{1, i-M} \\ 0 & 0 & \ddots & a_{M, i-M^{2}} \\ & \vdots & 0\end{array}\right]_{K \times K} \quad \begin{aligned} & i \in[0, L] \\ & \end{aligned}$

Note that in these matrices the elements $a_{p, q}$ 's are set to zero if either $p$ or $q$ goes outside the region $W$ and $a_{00}=$ -1 . As evident from the 2-D block recursive equation (8), the current diagonal block $X_{i, j}$ can be evaluated from the past three blocks $X_{i-1, j}, X_{i, j-1}, X_{i-1, j+1}$ and a block of driving noise sequence $U_{i, j}$. Fig. 2 illustrates the diagonal block processing and the formation of the processing window $\boldsymbol{P}$. In this figure a second-order AR model with NSHP region of support is arranged in a diagonal block form of length 3 and width 6 . As can readily be seen choosing blocks with smaller width than 6 in this case would lead to a block recursive equation with more terms on the past blocks. Note that if the block width is reduced to 1 , the block recursive equation will contain the same number of terms as in the scalar AR model. Now, if we define the local state vector in processing window $\boldsymbol{P}$ as

$$
S_{i, j}=\left[X_{i-1, j}^{t} X_{i-1, j+1}^{t} X_{i, j-1}^{t} X_{i, j}^{t}\right]^{t}
$$

then this local state can be expressed in terms of the past states. Using the block recursive equation (8) the following 2-D block state-space model can be obtained.

$$
S_{i, j}=A_{0,1} S_{i, j-1}+A_{1,-1} S_{i-1, j+1}+B U_{i, j} .
$$

The current block $X_{i, j}$ can be extracted from $S_{i, j}$ using

$$
X_{i, j}=C S_{i, j}
$$

where 


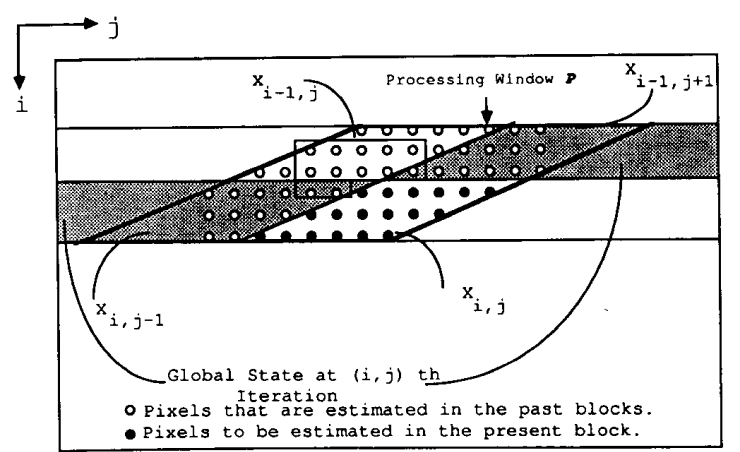

Fig. 2. 2-D diagonal block processing.
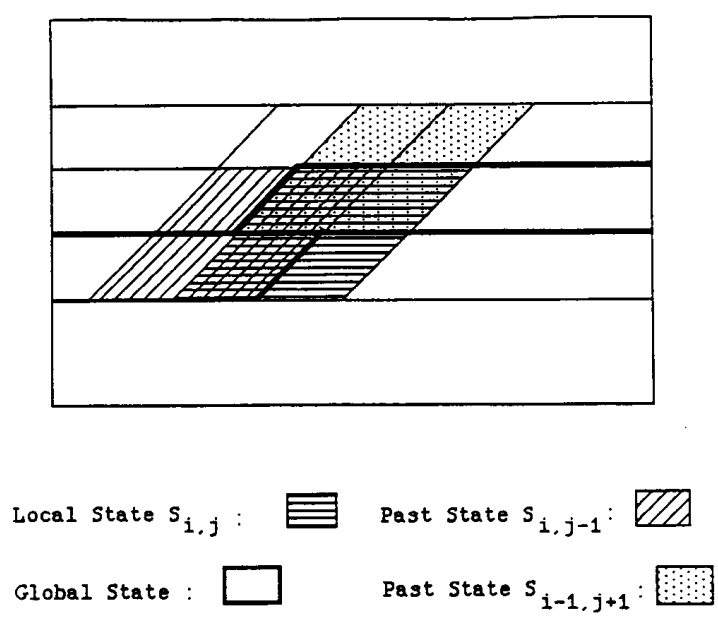

Fig. 3, Local and global state vectors when processing $(i, j)$ the block.

$$
\begin{aligned}
A_{0,1} & =\left[\begin{array}{llll}
0 & I & 0 & 0 \\
0 & 0 & 0 & 0 \\
0 & 0 & 0 & I \\
0 & B_{10} & 0 & B_{01}
\end{array}\right], \\
A_{1,-1} & =\left[\begin{array}{llll}
0 & 0 & 0 & 0 \\
0 & 0 & 0 & I \\
0 & 0 & 0 & 0 \\
0 & 0 & 0 & B_{1-1}
\end{array}\right] \\
B & =\left[\begin{array}{l}
0 \\
0 \\
0 \\
B_{00}
\end{array}\right], \quad \text { and } C=\left[\begin{array}{llll}
0 & 0 & 0 & I
\end{array}\right] .
\end{aligned}
$$

As can be observed from this state-space model the current local state, $S_{i, j}$, consists of four blocks. The first three blocks in $S_{i, j}$ are obtained from two blocks of the previous state vector $S_{i, j-1}$ and one block of the old state vector $S_{i-1, j+1}$ by shifting and data extraction (see Fig. 3 ). The last block of $S_{i, j}$, i.e., $X_{i, j}$, is evaluated from these three blocks and a block of driving noise, $U_{i, j}$, via the 2-D block recursive equation $(8 \mathrm{~b})$. The state vector $S_{i, j}$ propagates in two directions; the lower and upper right blocks are needed when the processing is moved to the next processing window; the lower left block is needed when the processing is moved to the next strip. As a result, the local state at location $(i, j)$ only consists of $S_{i, j-1}$ and $S_{i-1, j+1}$ state vectors whereas the global state at this location includes all $(Q+1)$ blocks $S_{i-1, k}, k \in[j, Q]$ and $S_{i, l}, l \in[0, j-1], Q:=(\lceil N / L\rceil+1)$ on the two adjacent strips. A pointer is used to keep track of the head of the global state vector. This process is known as reduced updating of the global state vector [11]. The local and global states are shown in Fig. 3 .

\section{Observation Model}

The image generated by the SAR is actually an image with complex values. The recorded intensity image is equivalent to a coherent image passed through a squarelaw detector. The coherent image may be considered a reflectance image multiplied by a Gaussian probability distribution linearly transformed by passing through a lowpass filter whose bandwidth is inversely proportional to the resolution [3]. The Gaussian distribution results from the central-limit theorem and the fact that there are many independent scatterers. The square-law detector changes this Gaussian probability distribution to a negative exponential distribution which has been shown to be reasonable for fully developed speckle. For this negative exponential distribution the mean is equal to the standard deviation. This fact indicates the multiplicative nature of the speckle, that is, the higher the average intensity, the noisier the area appears. In order to assure that the statistics of the polarized components of the detected intensity have a negative exponential distribution and that the speckle be modeled by a multiplicative noise, we assume that:

(a) The object is sufficiently rough compared to the radar wavelength to produce fully developed speckle in the image plane [3];

(b) There is a sufficiently large number of independent scatterers within one resolution cell [2], [3];

(c) The elements of the detector array are small compared to the speckle size [1].

With the above assumptions the recorded image in presence of both multiplicative speckle noise and additive thermal noise can be modeled as

$$
y(m, n)=[\gamma(m, n) \cdot x(m, n)]^{* *} h(m, n)+v(m, n)
$$

where in this case $h(m, n)$ is the point spread function (PSF) of the radar system; $\gamma(m, n)$ is a scalar white sequence with nonzero mean $\mu_{\gamma}$ and variance $\sigma_{\gamma}^{2}$ which represents the speckle noise; $v(m, n)$ is a scalar white noise sequence with zero mean and variance $\sigma_{v}^{2}$ which repre- 
sents additive thermal noise; $x(m, n)$ is the desired uncorrupted image; $y(m, n)$ is the corrupted recorded image and ** represents 2-D linear convolution operation. If we assume that the support region of the PSF is limited to $\bar{W}$, $\bar{W}=W \cup(0,0)$ the observation model in block form becomes

$$
Y_{i, j}=H \Gamma_{i, j} S_{i, j}+V_{i, j}
$$

where $H$ is given by

$$
H=\left[H_{10} H_{1-1} H_{01} H_{00}\right]
$$

and matrices $H_{i j}$ 's are block Toeplitz matrices with Toeplitz blocks defined in terms of $h(m, n)$ similar to $A_{i j}$ 's in (9) and (10); $\Gamma_{i, j}$ is a diagonal matrix consisting of speckle noise samples $\gamma(m, n)$ within one window forming from four blocks of data; $V_{i, j}$ represents a block of additive white noise $v(m, n)$; and finally $Y_{i, j}$ is a block of received (degraded) image.

In what follows the block equations (12) and (14) for the image and the degradation processes are combined to form the complete dynamic equation needed for the Kalman filtering. Then the relevant 2-D BKF equations are derived.

\section{Block Dynamic Model and Kalman Filter EQUATIONS}

Combining the image generation model in (12) and the observation model in (14) would yield a complete block dynamic model with uncertain observation [26], [27] due to the presence of the multiplicative noise. This 2-D block state-space dynamic model is

$$
\begin{aligned}
& S_{i, j}=A_{0,1} S_{i, j-1}+A_{1,-1} S_{i-1, j+1}+B U_{i, j} \\
& Y_{i, j}=H \Gamma_{i, j} S_{i, j}+V_{i, j}
\end{aligned}
$$

where

$$
\begin{aligned}
E\left[V_{i, j}\right] & =\mathbf{0} \\
E\left[\Gamma_{i, j}\right] & =\mu_{\gamma} I \\
E\left[V_{i, j} V_{i-m, j-n}^{t}\right] & =\boldsymbol{\sigma}_{\imath}^{2} I \boldsymbol{\delta}(m, n) \\
E\left[\Gamma_{i, j} \Gamma_{i-m, j-n}^{t}\right] & =\boldsymbol{\sigma}_{\gamma}^{2} I \boldsymbol{\delta}(m, n)+\mu_{\gamma}^{2} I \\
E\left[U_{i, j} U_{i-m, j-n}^{t}\right] & =\boldsymbol{\sigma}_{u}^{2} I \boldsymbol{\delta}(m, n) .
\end{aligned}
$$

The 2-D Kalman filter equations for the model in (15) are derived as

$$
\begin{aligned}
\hat{S}_{i, j}= & A_{0,1} \hat{\hat{S}}_{i, j-1}+A_{1,-1} \hat{S}_{i-1, j+1} \\
P_{c}(i, j):= & E\left[\left(S_{i, j-1}-\hat{\hat{S}}_{i, j-1}\right)\left(S_{i-1, j+1}-\hat{S}_{i-1, j+1}\right)^{t}\right] \\
= & {\left[I-\mu_{\gamma} K_{i, j-1} H\right] A_{1,-1} P_{a}(i-1, j) } \\
& \cdot A_{0,1}^{t}\left[I-\mu_{\gamma} K_{i-1, j+1} H\right]^{t} \\
P_{b}(i, j):= & E\left[\left(S_{i, j}-\hat{S}_{i, j}\right)\left(S_{i, j}-\hat{S}_{i, j}\right)^{t}\right] \\
= & A_{0,1} P_{a}(i, j-1) A_{0,1}^{t} \\
& +A_{1,-1} P_{a}(i-1, j+1) A_{1,-1}^{t}
\end{aligned}
$$

$$
\begin{aligned}
& +A_{0,1} P_{c}(i, j) A_{1,-1}^{t} \\
& +A_{1,-1} P_{c}(i, j)^{t} A_{0,1}^{t}+B B^{t} \sigma_{u}^{2} \\
K_{i, j}= & \mu_{\gamma} P_{b}(i, j) H^{t} R_{Z}^{-1}(i, j) \\
\hat{S}_{i, j}= & \hat{S}_{i, j}+K_{i, j} Z_{i, j} \\
P_{a}(i, j):= & E\left[\left(S_{i, j}-\hat{S}_{i, j}\right)\left(S_{i, j}-\hat{S}_{i, j}\right)^{t}\right] \\
= & {\left[I-\mu_{\gamma} K_{i, j} H\right] P_{b}(i, j) } \\
\hat{\hat{X}}_{i, j}= & C \hat{\hat{S}}_{i, j}
\end{aligned}
$$

where $\hat{S}_{i, j}$ and $\hat{\hat{S}}_{i, j}$ are, respectively, the a priori (before the updating) and the a posteriori (after the updating) suboptimal estimates of $S_{i, j} ; Z_{i, j}$ is the innovation sequence that is, $Z_{i, j}=Y_{i, j}-\mu_{\gamma} H S_{i, j}$ with zero mean and correlation matrix

$$
E\left[Z_{i, j} Z_{i, j}^{\prime}\right]=R_{Z}(i, j)
$$

which is given by

$$
\begin{aligned}
R_{Z}(i, j)= & \mu_{\gamma}^{2} H P_{b}(i, j) H^{t}+\sigma_{\gamma}^{2}\left[\sigma_{x}^{2}(i, j)\right. \\
& \left.+\mu_{x}^{2}(i, j)\right] H H^{t}+\sigma_{p}^{2} I
\end{aligned}
$$

$P_{b}(i, j)$ and $P_{a}(i, j)$ are the $a$ priori and the a posteriori error covariance matrices, respectively; $P_{\iota}(i, j)$ is the error cross-covariance matrix; $K_{i, j}$ is the Kalman gain matrix; and $\sigma_{x}^{2}(i, j)$ and $\mu_{x}(i, j)$ represent the local variance and the local mean of the original image in the $(i, j)$ th block, respectively.

\section{Remarks}

1. For the 2-D state-space model (15) to be BIBO stable, it is necessary that all the eigenvalues of matrices $A_{0,1}$ and $A_{1,-1}$ be in the interior of the unit circle [28].

2. The eigenvalues of matrix $\left[I-\mu_{\gamma} K_{i, j} H\right], \forall i, j$ must be in interior of the unit circle in order to have an asymptotically stable filter [29].

3 . The error covariances are decaying functions in distance.

4. As a consequence of remarks (1)-(3), all the higher order terms in the a priori error covariance expression $(17 \mathrm{c})$ are neglected. As a matter of fact, our simulation results indicate that the cross term $P_{c}(i, j)$ is also considerably small in comparison with the first two terms in $P_{b}(i$, $j$ ) and thus may be neglected.

5. In order to evaluate $\hat{S}_{i, j}$ in (17a) we need to keep the updated estimates of the past local states, $\hat{\hat{S}}_{i, j-1}$ and $\hat{S}_{i-1, j+1}$, in the global state which consists of all the $(Q$ $+1)$ state vectors $\hat{\hat{S}}_{i-1, k}, k \in[j, Q]$ and $\hat{\hat{S}}_{i, l}, l \in[0, j-$ 1] in two adjacent strips. Similarly, in order to compute $P_{c}(i, j)$ and $P_{b}(i, j)$ in $(17 \mathrm{~b})$ and $(17 \mathrm{c})$ we need to store all the past $P_{a}$ 's and $K$ 's namely $P_{a}(i, j-1), P_{a}(i-1$, $j), P_{a}(i-1, j+1), K_{i, j-1}$ and $K_{i-1, j+1}$ in the global storage for these matrices. The storage of $K$ 's may be avoided if in the computation of $P_{b}(i, j)$ the terms asso- 
ciated with $P_{c}(i, j)$ are neglected. To avoid saving all these states and matrices, a new Kalman filtering scheme is suggested in [30] which requires only the local states and local $P_{a}$ and $K$ matrices.

6. In the above Kalman filtering equations the assumption is that the image is stationary within one window (small region). The term $\left[\sigma_{x}^{2}(i, j)+\mu_{x}^{2}(i, j)\right]$ in (19) for the special case when $H=\left[\begin{array}{llll}0 & 0 & 0 & I\end{array}\right]$, i.e., no blur, can be obtained from the local statistics in the $(i, j)$ th block of the observed image. The covariance matrix for the observed image in the $(i, j)$ th block is

$$
\begin{aligned}
C_{Y}(i, j):= & \left(Y_{i, j}-\bar{Y}_{i, j}\right)\left(Y_{i, j}-\bar{Y}_{i, j}\right)^{t} \\
= & {\left[H\left(\boldsymbol{\Gamma}_{i, j} S_{i, j}-\mu_{\gamma} \bar{S}_{i, j}\right)+V_{i, j}\right] } \\
& \cdot\left[\left(S_{i, j}^{t} \boldsymbol{\Gamma}_{i, j}^{t}-\mu_{\gamma} \bar{S}_{i, j}\right) H^{t}+V_{i, j}^{t}\right] \\
= & H\left(\boldsymbol{\Gamma}_{i, j} S_{i, j} S_{i, j}^{t} \Gamma_{i, j}^{t}-\mu_{\gamma}^{2} \bar{S}_{i, j}^{2}\right) H^{t}+V_{i, j} V_{i, j}^{t},
\end{aligned}
$$

where "-, represents the mean of the relevant vector. Taking the trace of both sides and dividing by KL yields

$$
\boldsymbol{\sigma}_{y}^{2}(i, j)=\boldsymbol{\sigma}_{\gamma}^{2} \mu_{x}^{2}(i, j)+\left(\boldsymbol{\sigma}_{y}^{2}+\mu_{\gamma}^{2}\right) \boldsymbol{\sigma}_{x}^{2}(i, j)+\boldsymbol{\sigma}_{i}^{2} .
$$

Note that

$$
\mu_{x}(i, j)=\mu_{y}(i, j) / \mu_{\gamma}
$$

where $\sigma_{y}^{2}(i, j)$ and $\mu_{y}(i, j)$ are the local variance and the local mean of the observed image in the $(i, j)$ th block, respectively. Knowing the statistics of the speckle noise, (21a) and (21b) can be used to find $\sigma_{x}^{2}(i, j)$ and $\mu_{x}(i, j)$. Note that for a one-look image the mean and variance of $\gamma(m, n)$ are unity [1].

The 2-D BKF equations in (17) provide an adaptive local estimator in which the Kalman gain matrix is adjusted based upon the local statistics within each block via (19) and (17d). If further improvement in the performance of the filter is required, the adaptation should also account for the changes in the AR model parameters in addition to those in (19). This topic is addressed in the following section.

\section{Image Model Parameter Identification}

In this section a new 2 -D recursive identification scheme for computing the AR model parameters at each stage of the algorithm is developed. This method which takes into account the effects of multiple degradations is inspired from the 1-D recursive prediction method in [31] and makes use of the stochastic Newton approach.

In order to facilitate the derivation we map the 2-D array $(i, j)$ to a 1-D array using the index mapping

$$
\begin{gathered}
k=i Q+j+1, \quad i \in[0,(\lceil N / K\rceil-1)], \\
j \in[0,(Q-1)] .
\end{gathered}
$$

Using this mapping we have

$$
\begin{aligned}
X_{i, j} \rightarrow X_{k} & X_{i, j-1} \rightarrow X_{k-1} \\
X_{i-1, j} \rightarrow X_{k-Q} & X_{i-1, j+1} \rightarrow X_{k-Q+1} .
\end{aligned}
$$

This applies to any other block vector in the model. Now, the 2-D block state-space equation (15) in space varying form which accounts for local changes in statistics within one window becomes

$$
\begin{aligned}
& S_{k}=A_{0.1}(\phi) S_{k-1}+A_{1,-1}(\phi) S_{k-Q+1}+B(\phi) U_{k} \\
& Y_{k}=H \Gamma_{k} S_{k}+V_{k}
\end{aligned}
$$

where the elements of the unknown parameter vector $\phi$ are $a_{i, j}$ 's arranged lexicographically. Now, use (17e) to transform the model into the "filtered form", i.e.

$$
\begin{aligned}
\hat{S}_{k} & =\hat{S}_{k}+K_{k}(\phi)\left[Y_{k}-\mu_{\gamma} H \hat{S}_{k}\right] \\
& =\left[I-\mu_{\gamma} K_{k}(\phi) H\right] \hat{S}_{k}+K_{k}(\phi) Y_{k} \\
\hat{Y}(k \mid \phi) & =\hat{Y}(k)=\mu_{\gamma} H \hat{S}_{k} .
\end{aligned}
$$

Given the above model the natural way to measure the goodness of the estimates is the prediction error $Z_{k}(\phi)$. A reasonable measure of the size of this vector is the quadratic norm

$$
N(\phi)=E\left[\frac{1}{2} Z_{k}^{\prime}(\phi) \Pi^{-1} Z_{k}(\phi)\right]
$$

where $\boldsymbol{I}$ is a positive definite matrix. The optimal choice of $\boldsymbol{I}$ is the covariance matrix of the true prediction error, i.e., $R_{Z}(k, \phi)$, which gives the smallest covariance matrix of the parameter estimates. The above quadratic criterion can be recursively minimized for $\phi$ using the stochastic Newton method [31]. This would lead to the following recursive identification step.

$$
\begin{aligned}
\phi(k)= & \phi(k-1)+v(k) \mathbb{R}^{-1}(k) \Delta(k, \phi) \\
& \cdot R_{Z}^{-1}(k, \phi) Z_{k}(\phi)
\end{aligned}
$$

where $R(k)$ is the Hessian (second derivative) of $N(\phi)$ wrt $\phi$ which can be computed recursively [31] using

$$
\begin{aligned}
\mathbb{R}(k)= & \mathbb{R}(k-1)+\mathbf{v}(k)\left[\mathbf{\Delta}(k, \phi) R_{Z}^{-1}(k, \phi) \mathbf{\Delta}^{\prime}(k, \phi)\right. \\
& -\mathbb{R}(k-1)] .
\end{aligned}
$$

In these equations $\{v(k)\}$ is the gain sequence which should satisfy

$\mathbf{v}(k) \geq 0, \quad \lim _{M_{0} \rightarrow \infty} \sum_{k=0}^{M_{o}} \mathbf{v}(k) \rightarrow \infty, \quad \lim _{M_{0} \rightarrow \infty} \sum_{k=0}^{M_{0}} v^{2}(k)<\infty$.

where $M_{o}$ is the number of blocks in the image. The third condition will ensure that the effects of noisy measurements are eliminated asymptotically; and the second condition guarantees the existence of a target. The choices for the gain sequence $\{v(k)\}$ and the initial value of $\mathscr{R}(k)$ are discussed later in this section. The gradient of the prediction is defined by

$$
\mathbf{\Delta}(k, \phi)=\left[\frac{d}{d \phi} \hat{Y}(k \mid \phi)\right]^{t} .
$$

Using (24b) we obtain

$$
\boldsymbol{\Delta}^{\prime}(k, \phi)=\mu_{\gamma} H \Sigma_{b}(k, \phi)
$$


where

$$
\boldsymbol{\Sigma}_{b}(k, \phi):=\frac{d}{d \phi} \hat{S}_{k}
$$

This can be computed recursively using [31]

$$
\begin{aligned}
\Sigma_{b}(k, \phi)= & A_{0,1}(\phi) \Sigma_{a}(k-1, \phi)+A_{1,-1}(\phi) \\
& \cdot \Sigma_{a}(k-Q+1, \phi)+W\left(\phi, \hat{S}_{k}\right)
\end{aligned}
$$

where

$$
W\left(\phi, \tilde{S}_{k}\right):=\frac{d}{d \phi}\left[A_{0,1}(\phi) \hat{\hat{S}}_{k-1}+A_{1,-1}(\phi) \hat{\hat{S}}_{k-Q+1}\right]_{\phi=\hat{\phi}(k)}
$$

and

$$
\boldsymbol{\Sigma}_{a}(k, \phi):=\frac{d}{d \phi} \hat{S}_{k}
$$

Matrix $\Sigma_{a}(k, \phi)$ can also be computed recursively by differentiating (24a) wrt $\phi$, i.e.

$$
\begin{aligned}
\Sigma_{a}(k, \phi) & =\frac{d}{d \phi}\left[\left(I-\mu_{\gamma} K_{k}(\phi) H\right) \hat{S}_{k}+K_{k}(\phi) Y_{k}\right] \\
& =\frac{d}{d \phi}\left[\hat{S}_{k}+K_{k}(\phi) Z_{k}(\phi)\right] \\
& =\left[I-\mu_{\gamma} K_{k}(\phi) H\right] \boldsymbol{\Sigma}_{b}(k, \phi)+\mathbf{\kappa} Z_{k}(\phi)
\end{aligned}
$$

where

$$
\boldsymbol{\kappa}:=\frac{d}{d \phi} K_{k}(\phi) .
$$

The components of the gradient matrix $\kappa$ can also be obtained [31] recursively by differentiating $(17 \mathrm{c})$ and $(17 \mathrm{~d})$ wrt $\phi$.

Having established the above results the recursive prediction algorithm becomes [20]

$$
\begin{aligned}
& Z_{k}(\phi)=Y_{k}-\hat{Y}_{k} \\
& \hat{S}_{k}=\hat{S}_{k}+K_{k}(\phi) Z_{k}(\phi) \\
& \Delta^{\prime}(k, \phi)=\mu_{\gamma} H \Sigma_{b}(k, \phi) \\
& \mathfrak{R}(k)=\mathfrak{R}(k-1)+\mathbf{v}(k)\left[\boldsymbol{\Delta}(k, \phi) R_{Z}^{-1}(k, \phi)\right. \\
& \text { - } \left.\boldsymbol{\Delta}^{t}(k, \phi)-\mathbb{R}(k-1)\right] \\
& \hat{\phi}(k)=\hat{\phi}(k-1)+v(k) R^{-1}(k) \Delta(k, \phi) \\
& \text { - } R_{Z}^{-1}(k, \phi) Z_{k}(\phi) \\
& W\left(\phi, \hat{S}_{k}\right):=\frac{d}{d \phi}\left[A_{0,1}(\phi) \hat{\hat{S}}_{k-1}\right. \\
& \left.+A_{1,-1}(\phi) \hat{\hat{S}}_{k-Q+1}\right]_{\phi=\hat{\phi}(k)} \\
& \Sigma_{a}(k, \phi)=\left[I-\mu_{\gamma} K_{k}(\phi) H\right] \Sigma_{b}(k, \phi)+\kappa Z_{k}(\phi)
\end{aligned}
$$

$$
\begin{aligned}
\mathbf{\Sigma}_{b}(k+1, \phi)= & A_{0.1}(\phi) \mathbf{\Sigma}_{a}(k, \phi)+A_{1,-1}(\phi) \\
& \cdot \mathbf{\Sigma}_{a}(k-Q+2, \phi)+W\left(\phi, \hat{S}_{k}\right) .
\end{aligned}
$$

The matrix inversion lemma [31] can be used for efficient evaluation of $R^{-1}(k)$ in (34d). However, the inversion algorithm is sensitive to roundoff error which results in numerical problems especially for large dimension matrices (large block size). These problems can be circumvented if matrix factorization techniques such as Cholesky decomposition or U-D factorization are employed [31]. Due to the fact that elemental block matrices of $A_{0.1}(\phi), A_{1,-1}(\phi)$, and $B(\phi)$ are block Toeplitz with Toeplitz blocks the operations involving these matrices can be accomplished using fast convolution techniques [23].

The above steps for the general case are clearly more complex and time consuming. The main computational burden is in the calculation of the gradient of the Kalman gain. To reduce the order of the computations dramatically, steady-state Kalman gain, $K_{s}$, can be obtained and used throughout the identification process. The relevant equations in this case would essentially be the same with the exception that $\boldsymbol{\kappa}$ does not appear in these equations. Thus (34b) and (34g) become

$$
\begin{aligned}
\hat{S}_{k} & =\hat{S}_{k}+K_{s} Z_{k} \\
\boldsymbol{\Sigma}_{a}(k, \phi) & =\left[I-\mu_{\gamma} K_{s} H\right] \Sigma_{b}(k, \phi) .
\end{aligned}
$$

The gain sequence, $\{v(k)\}$, in the adaptation equation (34d) has a significant influence on both the transient behavior and the accuracy of the estimation. Let us define a new sequence, $\{\lambda(k)\}$, which is called "forgetting factor' '[31] as

$$
\lambda(k)=\frac{\mathbf{v}(k-1)[1-v(k)]}{v(k)}
$$

or

$$
v(k)=\frac{1}{1+\lambda(k) / v(k-1)}
$$

which grows exponentially to 1 . It is suggested [31] that for most practical situations where the order of the AR model is not high the following numerical method be used to generate $\{\lambda(k)\}$,

$$
\lambda(k)=0.99 \lambda(k-1)+0.01 \quad \lambda(0)=0.95 .
$$

For large order models, $\lambda(k)$ should grow more slowly to 1. The choice of the initial estimate of $\mathbb{R}(k)$ is also discussed in [31]. If no prior information about the variance of the output innovation and covariance of $\phi(0)$ is available the reasonable choice for $R(0)$ is found to be

$$
R(0)=\rho^{-1} I .
$$

Small values of $\rho$ result in slower convergence. This corresponds to the case when too much confidence is given to the initial estimates $\phi(0)$. 


\section{ImPlementation and Results}

In this section, the recursive parameter identification algorithm developed in the previous section is used in conjunction with the 2-D BKF to remove the effects of speckle and additive noise from SAR images. A comparison is made between this 2-D ABKF and the local linear minimum variance estimator (LLMVE) in [13], [14], and [18].

For an image corrupted by both multiplicative and additive degradations the scalar observation equation (13) becomes

$$
y(m, n)=\gamma(m, n) x(m, n)+v(m, n) .
$$

The filtering equation using LLMVE is

$$
\begin{aligned}
\hat{x}(m, n)= & \mu_{x}(m, n)+k(m, n) \\
& \cdot\left[y(m, n)-\mu_{\gamma} \mu_{x}(m, n)-\mu_{r}\right]
\end{aligned}
$$

where the gain $k(m, n)$ is given [13] by

$$
k(m, n):=\mu_{\gamma} Q(m, n) /\left(\mu_{*}^{2}(m, n) \boldsymbol{\sigma}_{\gamma}^{2}+\mu_{\gamma}^{2} Q(m, n)+\boldsymbol{\sigma}_{l}^{2}\right)
$$

$$
Q(m, n):=\frac{\sigma_{y}^{2}(m, n)+\mu_{\gamma}^{2}(m, n)}{\sigma_{\gamma}^{2}+\mu_{\gamma}^{2}}-\mu_{x}^{2}(m, n)-\sigma_{l}^{2} .
$$

The statistics of the image are measured based upon the local information within each window of the observed image [13], [18] and using (21). For the case of multiplicative noise alone $\sigma_{r}^{2}=0$. In this case, using (21) and considering the fact that $\mu_{\gamma}=1$ we obtain

$$
Q(m, n)=\sigma_{x}^{2}(m, n)
$$

and thus

$$
k(m, n)=\boldsymbol{\sigma}_{x}^{2}(m, n) /\left(\mu_{x}^{2}(m, n) \boldsymbol{\sigma}_{y}^{2}+\sigma_{x}^{2}(m, n)\right)
$$

which is the result obtained in [14] and [18]. This result is essentially equivalent to the scalar version of the steady state non-adaptive block Kalman filter in (17e) and (19).

The test images used for our study are shown in Figs. 4( $a$ and $b)$ and 5. Fig. 4( $a$ and $b$ ) shows portions of the original SAR image obtained from the Jet Propulsion Laboratory (JPL) which contained complex field data for each pixel. These digitally correlated "farm" images have a resolution of $512 \times 512$ and the number of gray levels which is 256 . The images are obtained by mapping the complex data to the intensity image as opposed to the square root of the intensity because the square root operation changes the negative exponential distribution to the Rayleigh distribution, thus compressing the dynamic range of the SAR image and reducing the speckle standard deviation by approximately a factor of 2 . However, the intensity distribution has a long tail in the high end of the range with very low probability in this tail. As a result, a slight compression of the upper end of the scale had to be done to map those pixels that lie in this region into a small range of the gray levels (5 levels). This only affects the

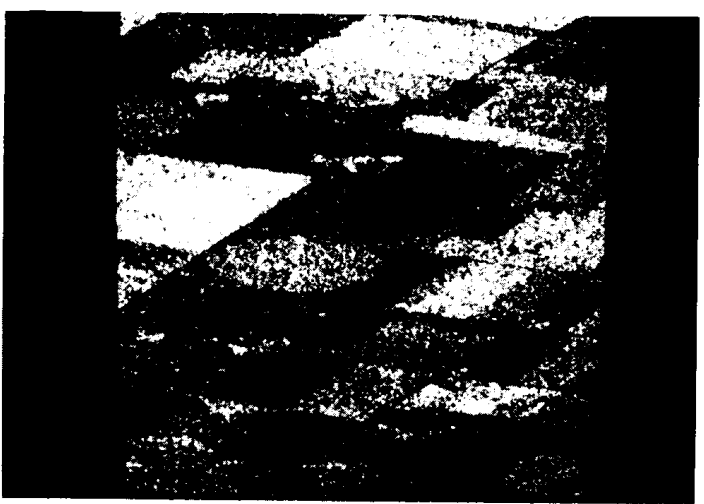

(a)

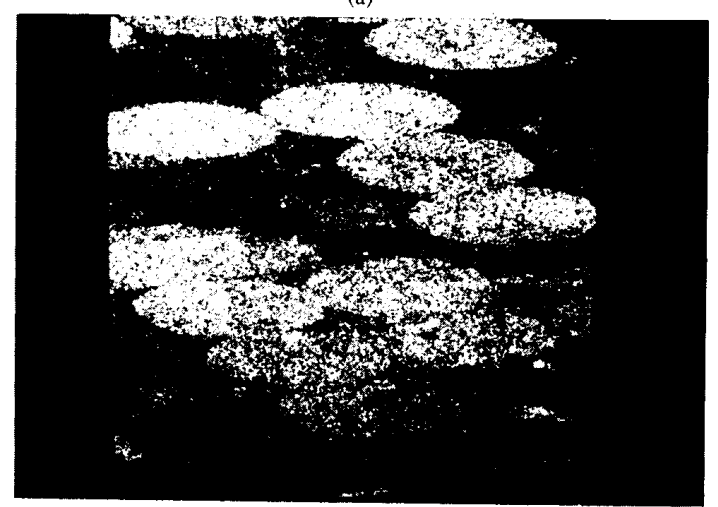

(b)

Fig. 4. (a) Original SAR image of Farm I (speckle only). (b) Origina SAR image of Farm 2 (speckle only).

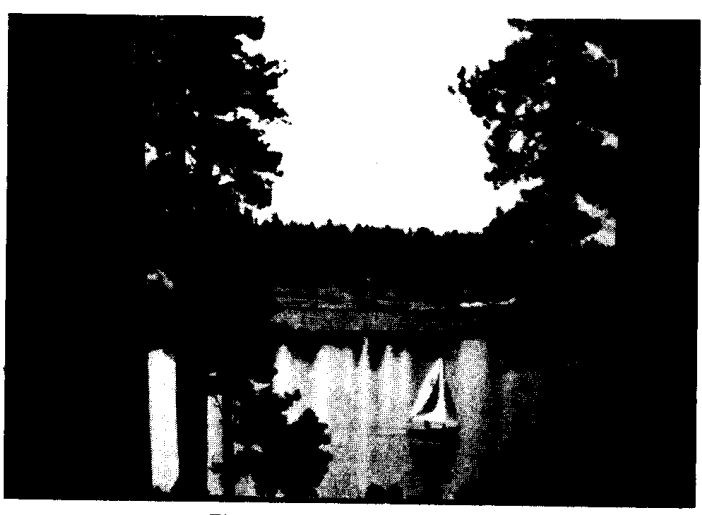

Fig. 5. Original boat image.

statistics of the images negligibly. The "boat" image in Fig. 5 is artificially corrupted by a multiplicative noise of Gaussian distribution with mean of $\mu_{y}=0.75$ and standard deviation of $\sigma_{\gamma}=0.25$. The resultant image is shown in Fig. 6. These images are used as inputs to the 2-D ABKF and LLMVE in order to study the performance of these filters in removing the speckle effects only.

The results of applying LLMVE in (39), (40), and (41) to these images are shown in Figs. 7( $a$ and $b)$ and 8 , re- 


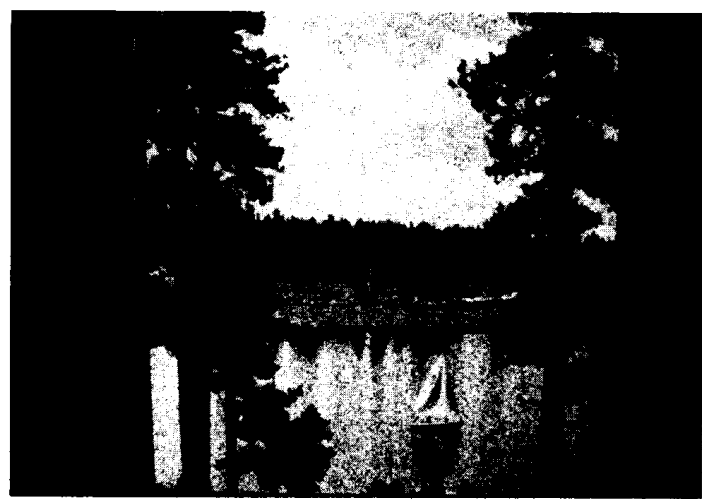

Fig. 6. Degraded boat image with multiplicative noise.
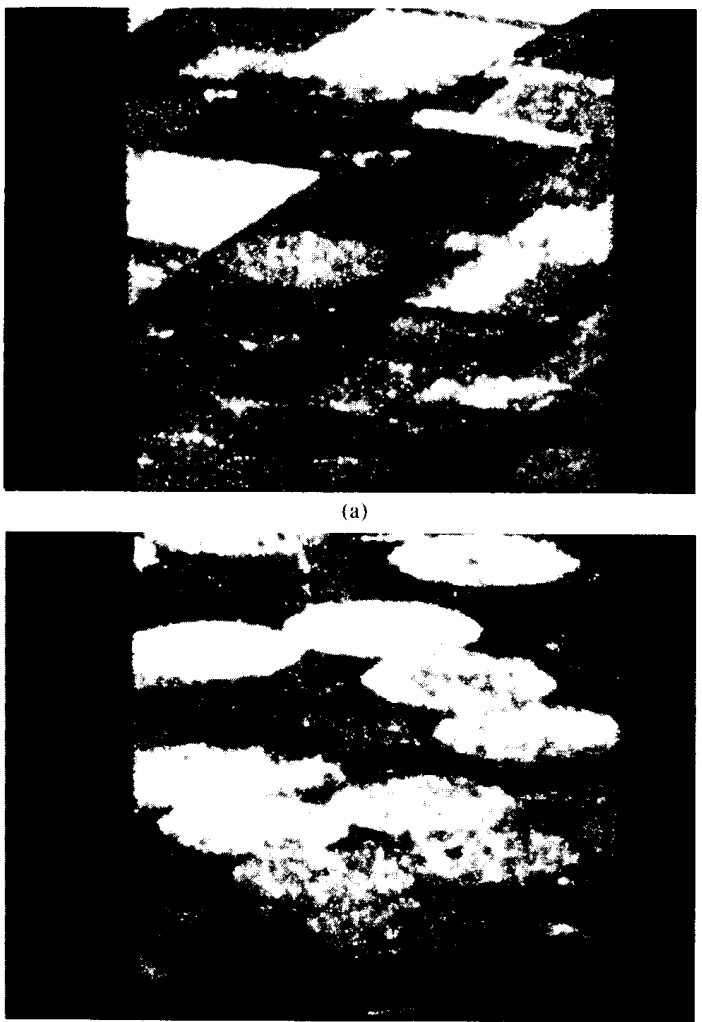

(b)

Fig. 7. (a) Processed Farm 1 image using LLMVE. (b) Processed Farm 2 image using LLMVE.

spectively. Processing windows of size $7 \times 7$ are used throughout this experiment. Comparison with the original images shows over-smoothing in the light areas within the images and also smearing effects of the sharp edges. In addition, an artifact similar to the "salt and pepper" noise is clearly evident in the processed images. The oversmoothing and smearing effects become even more prominent when the input images are corrupted by both mul-

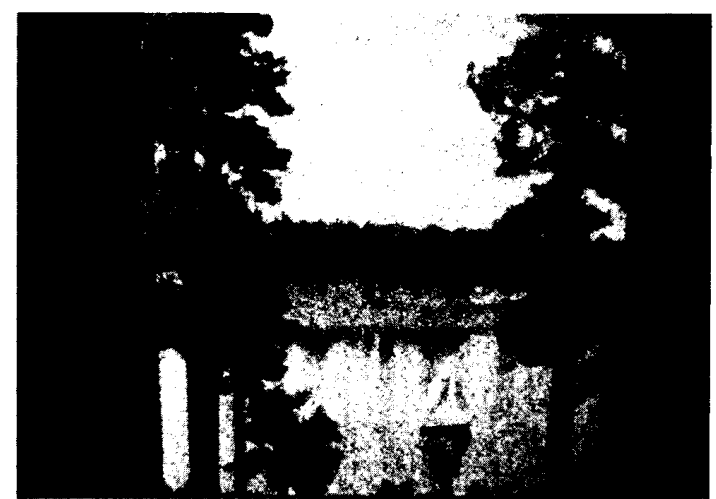

Fig. 8. Processed boat image (multiplicative noise) using LLMVE.

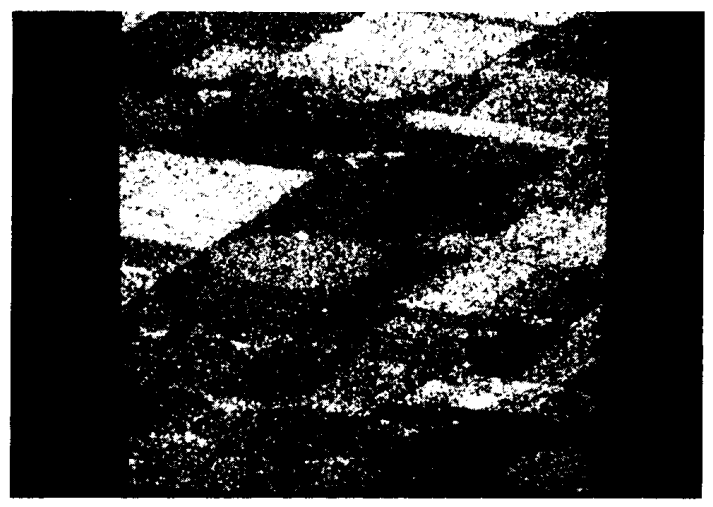

(a)

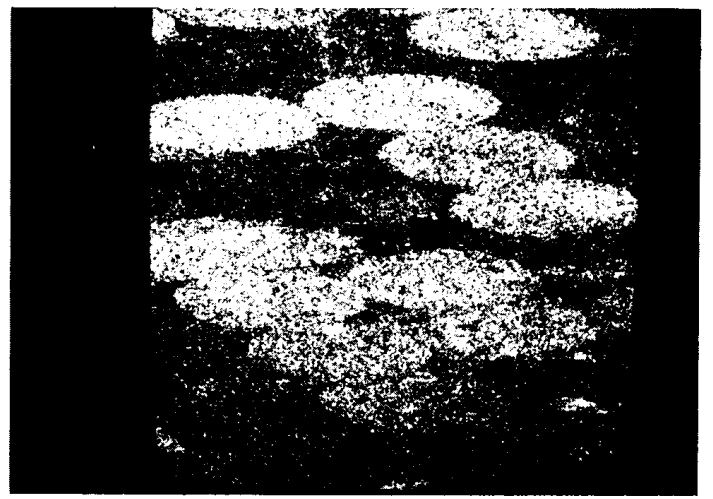

(b)

Fig. 9. (a) Farm 1 image degraded by both speckle and additive noise. (b) Farm 2 image degraded by both speckle and additive noise.

tiplicative and additive noises. Fig. 9(a and b) shows severely degraded images obtained by adding zero mean WG noise to the speckled images in Fig. 4( $a$ and $b$ ). The variance of the noise is chosen so that a SNR of $0 \mathrm{~dB}$ is achieved. The image in Fig. 10 is obtained by adding a zero mean WG to image in Fig. 6 to achieve a SNR of 5 $\mathrm{dB}$. The result of applying general LLMVE to the images in Figs. 9( $a$ and $b)$ and 10 are shown in Figs. $11(a$ and $b)$ 


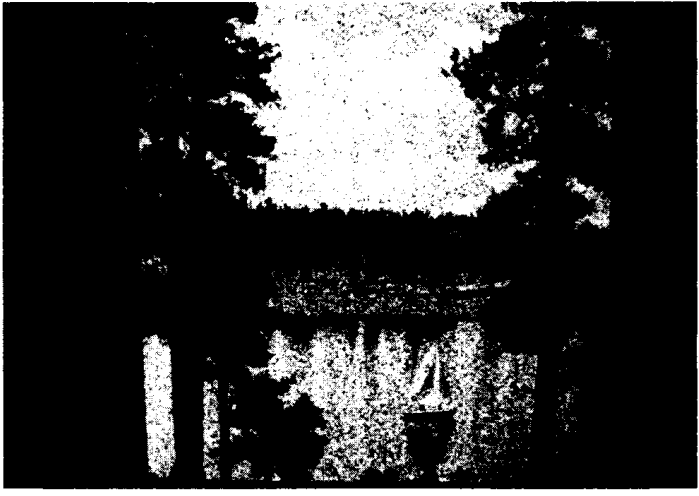

Fig. 10. Boat image degraded by both multiplicative and additive noises

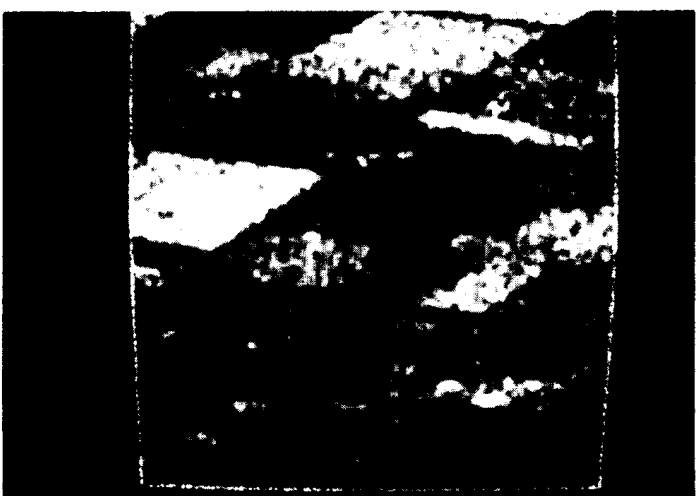

(a)

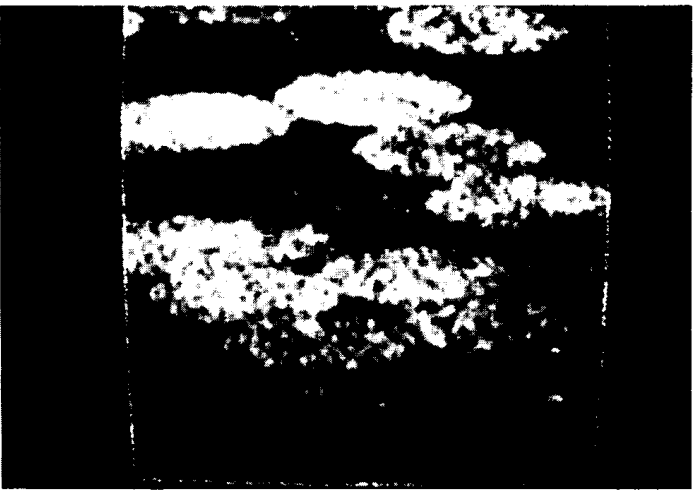

(b)

Fig. 11. (a) Processed Farm 1 image (speckle and additive noise) using LLMVE. (b) Processed Farm 2 image (speckle and additive noise) using LLMVE.

and 12 , respectively. The significant oversmoothing effect which has resulted in some loss of valid information is clearly visible in these images.

Next, the 2-D ABKF developed in this paper is used to remove the effects of the speckle and additive noise from the relevant test images. In this process the initial estimates for the model parameters are obtained based upon

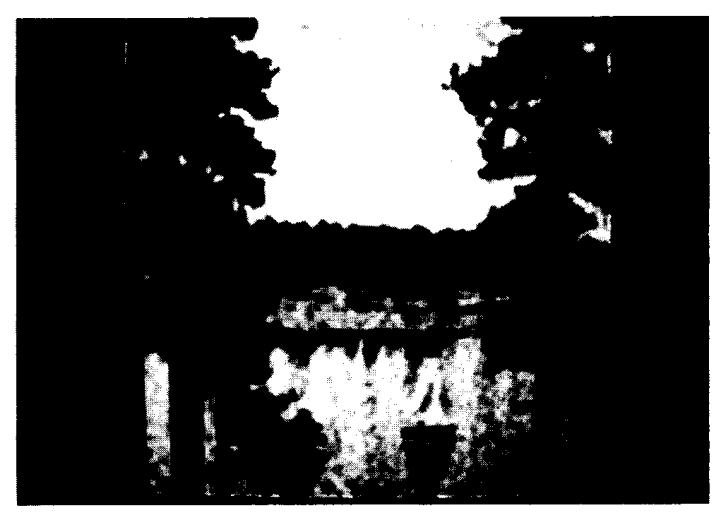

Fig. 12. Processed boat image (multiplicative and additive noises) using LLMVE.

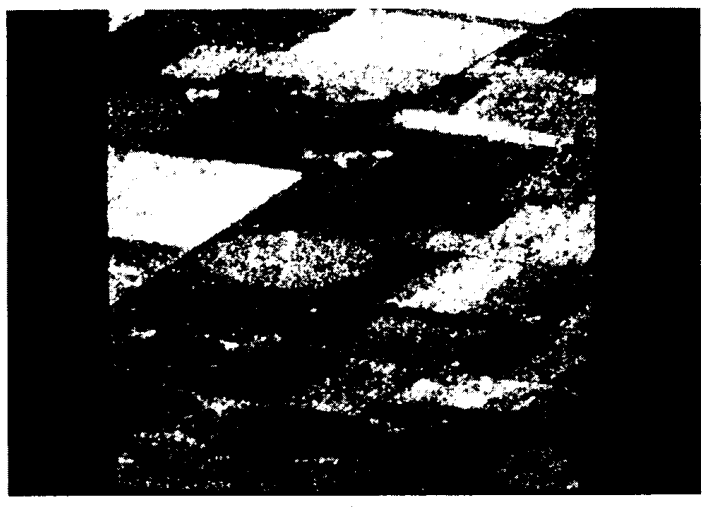

(a)

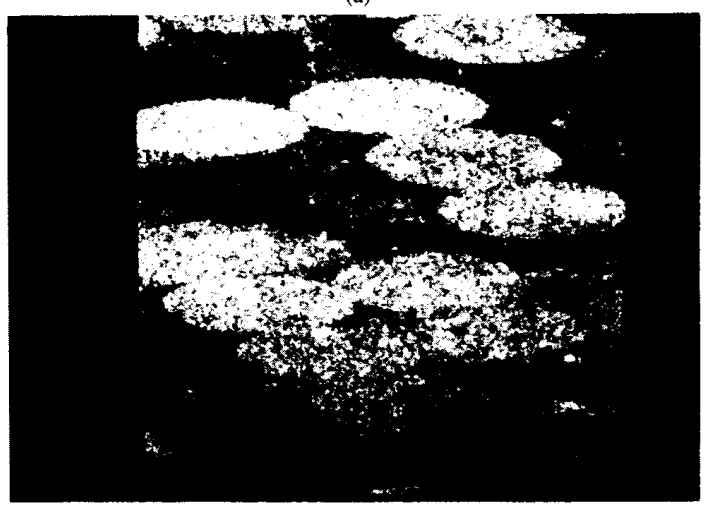

(b)

Fig. 13. (a) Processed Farm 1 image (speckle only) using 2-D ABKF. (b) Processed Farm 2 image (speckle only) using 2-D ABKF.

the global statistics of the degraded image. These parameters are iteratively updated for every block via the recursive identification scheme in (34) and (35). A second-order AR model is used to model the image process. Thus, the diagonal blocks are of size $3 \times 6$ and the effective correlation distance in a processing window is 6 in one direction and 18 in another. To reduce the computational 


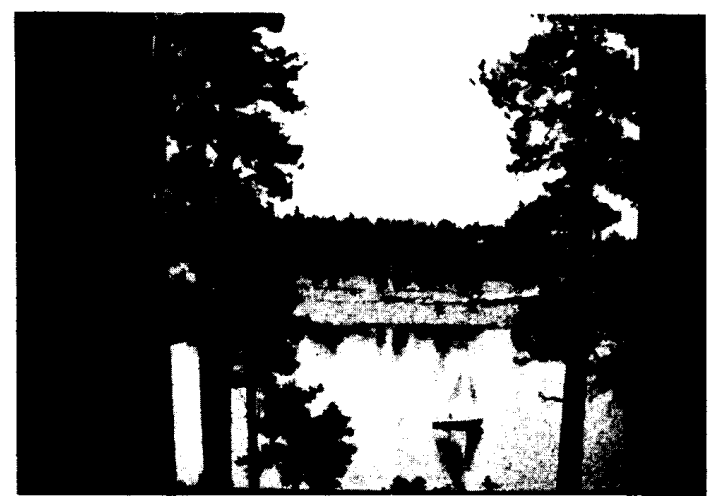

Fig. 14. Processed boat image (multiplicative noise) using 2-D ABKF.

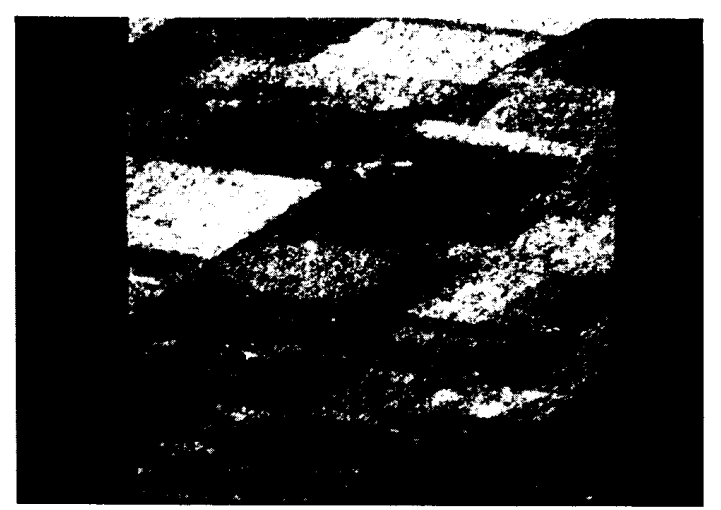

(a)

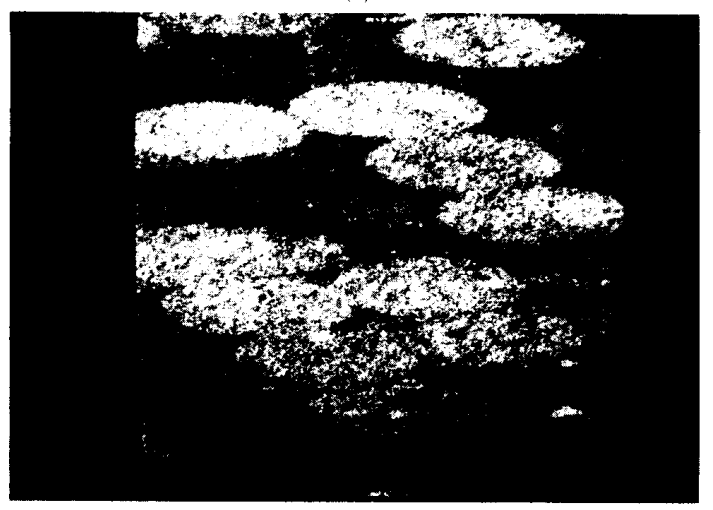

(b)

Fig. 15. (a) Processed Farm 1 image (speckle and additive noise) using 2 D ABKF. (b) Processed Farm 2 image (speckle and additive noise) using 2-D ABKF.

effort considerably a steady-state Kalman gain matrix is computed after approximately 15 iterations for the farm images and 20 iterations for the boat image. The steady state gains are then used throughout the entire processing. A reasonable choice for $R(0)$ based upon a trial and error procedure is found to be 10 for the farm images and 15

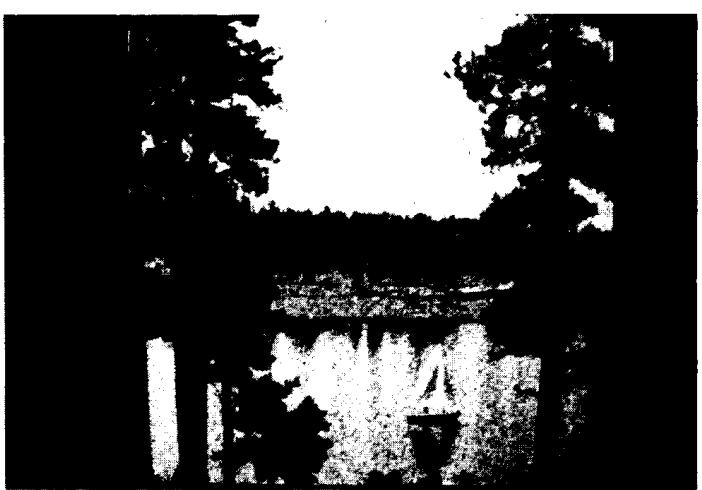

Fig. 16. Processed boat image (multiplicative and additive noises) using 2-D ABKF.

for the boat image. The results of applying 2-D ABKF to the images in Figs. 4( $a$ and $b), 6,9(a$ and $b)$, and 10 are shown in Figs. 13( $a$ and b), 14, 15( $a$ and b), and 16, respectively. As can be seen clearly the 2-D ABKF provide a compromise between the noise removal and the resultant smearing artifacts. That is, the results of the LLMVE indicate considerable smearing and some loss of valid information, although its noise removal capability is good. The results of the 2-D ABKF, on the other hand, show acceptable noise removal with very little smearing effects which is more pleasing to the human eyes. The CPU time for processing the entire image on a MicroVAX 3600 computer is found to be approximately $25 \mathrm{~min}$.

\section{CONCLUSION}

In this paper a new 2-D adaptive Kalman filter using the block-processing method is proposed for removing speckle and additive thermal noise from SAR imagery. A 2-D block state-space structure is derived when the image is modeled by an AR process with NSHP region of support. To avoid the noncausality problem which is caused by the direct use of the block processing, the pixels are arranged in a diagonal fashion forming a so-called " $\mathrm{di}$ agonal block" structure. To account for the multiplicative nature of the speckle noise a new modified Kalman filtering scheme is developed in which the state vector propagates in two directions. For the 2-D block state-space model a new recursive parameter identification scheme is proposed using the stochastic Newton approach. This algorithm can be implemented on-line to compute the AR model parameters for each new block of the image rather than each new pixel, hence reducing the number of iterations in the adaptation process. Using this method the image model parameters are adjusted based upon the local spatial activities within each processing window. As a result, the filtering operation would not perturb the edges significantly. Simulation results on various images are presented and a comparison with other local adaptive estimators is also provided. 


\section{REFERENCES}

[I] J. W. Goodman, "Some fundamental properties of speckle," J. Opt Soc. $A m$., vol. 66, pp. 1145-1150, Nov. 1976

[2] M. Tur, K. C. Chin, and J. W. Goodman, "When is speckle noise multiplicative?", Appl. Opt., vol. 21, pp. 1157-1159, Apr. 1982.

[3] G. April and H. H. Arsenault, "Nonstationary image-plane speckle statistes,"J. Opt. Soc. Am. A, vol. 1, pp. 738-741, July 1984

[4] H. H. Arsenault and G. April, "Properties of speckle integrated with a finite aperture and logarithmically transformed," J. Opt. Soc. Am. vol. 66, pp. 1160-1163, Nov. 1976

[5] K. Kondo, "Image restoration by Wiener filtering in the presence of signal-dependent noise," Appl. Opt., vol. 16, pp. 2554-2558, Sept. 1976.

[6] B. E. A. Saleh and M. Rabbani. "Linear filtering of speckled images," Optics Communications, vol. 35, no. 3, pp. 327-331, Dec. 1980.

[7] A. K. Jain and C. R. Christensen, "Digital processing of images in speckle noise," SPIE, vol. 243, pp. 46-50, 1980.

[8] D. T. Kuan et al., "Nonstationary 2-D recursive restoration of images with signal-dependent noise," Proc. of ICASSP'84, pp. 37.4.137.4.4. San Diego, CA, March 1984

[9] C. L. Anderson and A. N. Netravali, "Image restoration based on subjective criterian," IEEE Trans. Syst. Man Cybern., vol. SMC-6. pp. 845-853. Dec. 1976

[10] J. F. Abramatic and L. M. Silverman, "Nonlinear restoration of noisy images," IEEE Trans. Pattern Anal. Machine Intel., vol. PAMI-4, pp. 141-149, March 1982

[11] V. K. Ingle and J. W. Woods, "Multiple model recursive estimation of images," Proc. ICASSP'79, pp. 642-645, Washington. DC, Apr. 1979.

[12] S. A. Rajala and R. J. P. DeFigueiredo, "Adaptive nonlinear image restoration by a modified Kalman filtering approach, " IEEE Trans. Acoust. Speech Signal, Processing, vol. ASSP-29, pp. 1033-1042. Oct. 1981.

[13] J. S. Lee, "Digital image enhancement and noise filtering by use of local statistics," IEEE Trans Pattern Anal. Machine Intel., vol. PAMI-2, pp. 165-168, March 1980.

[14] J. S. Lee, "Speckle suppression and analysis for synthetic aperture radar images," Optical Engineering. vol. 25. pp. 636-643, May 1986.

[15] D. T. Kuan et al. "Adaptive noise smoothing filter for images with signal-dependent noise." IEEE Trans. Pattern Anal. Machine Intel. vol. PAMI-7, pp. 165-177, March 1985.

[16] V. S. Frost et al., "A model of radar images and its application of digital filtering of multiplicative noise," IEEE Trans. Pattern Anal Machine Intel., vol. PAMI-4, pp. 157-166, March 1982.

[17] J. W. Woods and J. Biemond, "Comments on a model for radar image and its application to adaptive digital filtering of multiplicative noise," IEEE Trans. Pattern Anal. Machine Intel., vol. PAMI-6, pp. 658-659, Sept. 1984

[18] D. T. Kuan et al. "Adaptive restoration of images with speckle," IEEE Trans. Acoust. Speech Signal Processing, vol. ASSP-35, pp 373-382, March 1987.

[19] M. R. Azimi-Sadjadi, "Speckled image restoration by adaptive block Kalman filtering," Proc. IEEE Int. Symp. on Geoscience and Remote Sensing (IGARS 87), pp. 1449-1455, Ann Arbor, MI, May 1987.

[20] M. R. Azimi-Sadjadi, "Two-Dimensional Adaptive Block Recursive Filtering," Proc. of Inter. Symp. on Circuits and Systems, (ISCAS 87), pp. 446-450, Philadelphia. PA. May 1987.

[21] S. Ranganath and A. K. Jain, "Two-Dimensional Linear Prediction Models-Part I: Spectral Factorization and Realization," IEEE Trans. Vol. ASSP-33, pp. 280-299, February 1985.

[22] R. A. King et al., Digital Filtering in One and Two Dimensions, Chapter 10. New York: Plenum Press, 1989.
[23] M. R. Azimi-Sadjadi and R. A. King, "Two dimensional block processors structures and implementation," IEEE Trans. Circ. Syst. vol. CAS-33, pp. 42-50, Jan. 1986.

[24] R. Gnanasekaran, "Block implementation of half-plane digital filters," IEEE Trans. Circ. Syst., vol. CAS-34, pp. 308-313, March 1987.

[25] J. H. Lee, "Two-dimensional block implementation of symmetric half-plane recursive digital filters," IEEE Trans. Circ. Syst., vol. CAS-35, pp. 736-742, June 1988.

[26] R. A. Monzingo, "Discrete optimal linear smoothing for systems with uncertain observations," IEEE Trans. Inform. Theory, vol. IT-21, pp. 271-275, May 1975

[27] P. K. Rajasekaran et al., "Optimum linear estimation of stochastic signals in the presence of multiplicative noise." IEEE Trans. Aerosp. Electron. Syst, vol. AES-7, pp. 462-468, May 1971.

[28] W. S. Lu and E. B. Lee, "Stability analysis for two-dimensional systems," IEEE Trans. Circ. Syst., vol. CAS-30, pp. 455-461, July 1983

[29] B. D. O. Anderson and J. B. Moore, Optimal Filtering. Englewood Cliffs. NJ: Prentice-Hall. 1979.

[30] S. Citrin and M. R. Azimi-Sadjadi, A Full-Plane Block Kalman Filter For Inage Estimation," to be submitted to IEEE Trans. Acoust. Speech Signal Processing.

[31] L. Ljung and T. Soderstrom. Theory and Practice of Recursive Identification. Cambridge, MA: MIT Press, 1983.

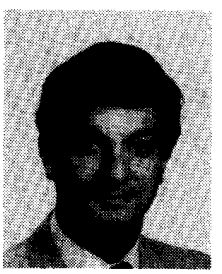

Mahmood R. Azimi-Sadjadi was born in Tehran, Iran, in 1952. He received the B.S. degree from the University of Tehran, Iran, in 1977, and the M.S. and Ph.D. degrees from Imperial College, University of London, England, in 1978 and 1982, respectively, all in electrical engineering.

He served as an assistant professor in the Department of Electrical and Computer Engineering. University of Michigan-Dearborn. Since July 1986 he has been with the Department of Electrical Engineering, Colorado State University, where he is now an associate professor. His areas of interest are digital signal/image processing, multidimensional system theory and analysis, adaptive filtering, system identification, and neural networks. He is a co-author of the book Digital Filtering in One and Two Dimensions, Plenum Press, 1989.

Dr. Azimi-Sadjadi is the recipient of the 1990 BATTELLE Summer Faculty Fellowship award, and the 1984 DOW Chemical Outstanding Young Faculty Award of the American Society for Engineering Education.

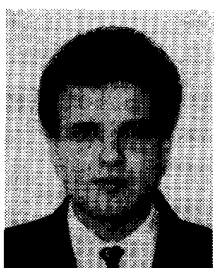

Sami Bannour was born on July 4, 1968 in Tunis, Tunisia. He received the B.Sc (Honors) degree in electrical engineering from Colorado State University in 1989. Since 1989, he has been working towards the M.Sc degree at Colorado State University while serving as a teaching and research assistant in the Department of Electrical Engineering.

His current research interests include statistical signal and image processing, and neural networks. 